\title{
ESTIMATES FOR SOLUTIONS TO THE TRANSPORT EQUATION UNDER THE PERTURBATION OF ITS ATTENUATION AND SCATTERING TERMS
}

\author{
DAIKI TANAKA, NOBUYUKI HIGASHIMORI AND YUUSUKE ISO
}

\begin{abstract}
The aim of the paper is to give $L^{p}$-estimates for the weak solution to the transport equation with $L^{\infty}$-perturbation both of the attenuation coefficient and of the scattering kernel. We try to clarify the constants in our estimates, and we show the estimates by a direct calculation. We introduce the albedo operator for the solutions, and we show stability of perturbed albedo operators.
\end{abstract}

\section{Introduction}

The transport equation is one of the mathematical models to support the diffuse optical tomography (DOT) (e.g. [2], [3], [7],[8] etc.), which is considered as a coming medical tomography. We give $L^{p}$-estimates to solutions to the equation as a basic study of DOT.

The governing equation of light propagation in biomedical tissues is described by the transport equation as a mathematical model, and it is well known that the solutions to the transport equation are approximated by those to the diffusion equation (e.g. [3]). DOT is reduced, from a theoretical viewpoint, to an inverse problem of the transport equation or of the diffusion equation, and the authors consider the former approach is more suitable to realize high-resolution DOT. We regard DOT as the inverse problem to determine the unknown attenuation coefficient in the transport equation ([3] etc.), and we show some estimates, which are expected to give so-called a priori information in the analysis of the inverse problem. More in detail, we estimate the $L^{p}$-weak solutions to the transport equation in the case that the attenuation coefficient and/or the scattering kernel are perturbed.

Let $T$ be a positive number, let $\Omega$ be a domain of $\mathbb{R}_{x}^{3}$ with a smooth boundary $\partial \Omega$, and let $U$ be a bounded domain of $\mathbb{R}_{\xi}^{3}$. We set

$$
\begin{aligned}
& Q_{T}:=(0, T) \times \Omega \times U, \\
& \Gamma_{ \pm}:=\left\{(t, x, \xi) \in \overline{Q_{T}} \mid x \in \partial \Omega \text { and } \pm n(x) \cdot \xi>0\right\},
\end{aligned}
$$

where $n(x)$ is the unit outer normal vector at $x \in \partial \Omega$. We consider the initial-boundary value

Corresponding author: Daiki Tanaka.

2010 Mathematics Subject Classification. 35Q60, 35R09.

Key words and phrases. Transport equation, stability estimate for perturbation, albedo operator, optical tomography. 
problem of the transport equation:

$$
\begin{aligned}
\left(\frac{\partial}{\partial t}+\xi \cdot \nabla_{x}\right) u(t, x, \xi) & =-\mu(t, x, \xi) u(t, x, \xi)+\int_{U} k\left(t, x, \xi, \xi^{\prime}\right) u\left(t, x, \xi^{\prime}\right) d \xi^{\prime}, & (t, x, \xi) \in Q_{T}, \\
u(0, x, \xi) & =u_{0}(x, \xi), & x \in \Omega, \xi \in U, \\
u(t, x, \xi) & =q(t, x, \xi), & (t, x, \xi) \in \Gamma_{-},
\end{aligned}
$$

and the aim of the paper is to give estimates of solutions for the case that the attenuation coefficient $\mu$ and the scattering kernel $k$ are perturbed in $L^{\infty}$ sense.

Prior to the discussion, we should define the $L^{p}$-weak solution to (1.1)-(1.3). Let us assume $\mu(t, x, \xi) \in L^{\infty}\left(Q_{T}\right)$ and $k\left(t, x, \xi, \xi^{\prime}\right) \in L^{\infty}\left(Q_{T} \times U\right)$. We call a function $u(t, x, \xi) \in L^{p}\left(Q_{T}\right)$ $(1 \leq p<\infty)$ a weak solution to (1.1)-(1.3), when $u$ satisfies

$$
\begin{aligned}
\int_{Q_{T}} u\left(\frac{\partial}{\partial t}+\xi \cdot \nabla_{x}\right) \varphi d t d x d \xi+\int_{Q_{T}}\left(\int_{U} k u d \xi^{\prime}-\mu u\right) \varphi d t d x d \xi & \\
& =\int_{\Gamma_{-}} n(x) \cdot \xi q \varphi d t d v_{x} d \xi-\left.\int_{\Omega} u_{0} \varphi\right|_{t=0} d x d \xi
\end{aligned}
$$

for all $\varphi \in V:=\left\{\varphi \in C_{0}^{\infty}\left(\overline{Q_{T}}\right)|\varphi|_{\Gamma_{+}}=0\right.$ and $\left.\varphi(T, \cdot, \cdot)=0\right\}$, where $d v_{x}$ denotes the surface element along $\partial \Omega$. Let us introduce the following notation. For $(t, x, \xi) \in \overline{Q_{T}}$, the line $l$ given by

$$
l:=\{(t+s, x+s \xi, \xi) \mid s \in \mathbb{R}\}
$$

is called a characteristic line of the transport equation (1.1), and we set $l_{ \pm}(t, x, \xi)$ and $l_{0}(t, x, \xi)$ by

$$
\begin{aligned}
l_{ \pm}(t, x, \xi) & :=\inf \left\{s \geq 0 \mid(t \pm s, x \pm s \xi, \xi) \notin Q_{T}\right\} \\
l_{0}(t, x, \xi) & :=l_{-}(t, x, \xi)+l_{+}(t, x, \xi) .
\end{aligned}
$$

We note $0 \leq l_{-}(t, x, \xi) \leq t \leq T$. We introduce weighted function spaces:

$$
\begin{aligned}
L^{p}\left(\Omega \times U ; l_{0}\right) & :=\left\{v: \Omega \times\left. U \rightarrow \mathbb{R}\left|\int_{\Omega \times U}\right| v(x, \xi)\right|^{p} l_{0}(0, x, \xi) d x d \xi<\infty\right\}, \\
L^{p}\left(\Gamma_{-} ; \rho_{-}\right) & :=\left\{q:\left.\Gamma_{-} \rightarrow \mathbb{R}\left|\int_{\Gamma_{-}}\right| q(t, x, \xi)\right|^{p} \rho_{-}(t, x, \xi) d t d v_{x} d \xi<\infty\right\},
\end{aligned}
$$

where $\rho_{-}(t, x, \xi):=l_{0}(t, x, \xi)|n(x) \cdot \xi|$ for $(t, x, \xi) \in \Gamma_{-}$. It is easily checked that these spaces are Banach spaces with the norms

$$
\begin{aligned}
\|v\|_{L^{p}\left(\Omega \times U ; l_{0}\right)} & :=\left(\int_{\Omega \times U}|v(x, \xi)|^{p} l_{0}(0, x, \xi) d x d \xi\right)^{\frac{1}{p}}, \\
\|q\|_{L^{p}\left(\Gamma_{-} ; \rho_{-}\right)} & :=\left(\int_{\Gamma_{-}}|q(t, x, \xi)|^{p} \rho_{-}(t, x, \xi) d t d v_{x} d \xi\right)^{\frac{1}{p}}
\end{aligned}
$$

respectively. Under the preparations above, we state the results about well-posedness of (1.1)(1.3), which are well-known ([5], [11]). 
Proposition 1.1. For $u_{0} \in L^{p}\left(\Omega \times U ; l_{0}\right)$ and $q \in L^{p}\left(\Gamma_{-} ; \rho_{-}\right)$, there exists a unique weak solution $u(t, x, \xi) \in L^{p}\left(Q_{T}\right)$ to $(1.1)-(1.3)$.

Proposition 1.2. Let $K$ be

$$
K:=\|\mu\|_{L^{\infty}\left(Q_{T}\right)}+|U|\|k\|_{L^{\infty}\left(Q_{T} \times U\right)},
$$

where $|U|$ denotes the Lebesgue measure of $U$. We have, if $u_{0} \in L^{p}\left(\Omega \times U ; l_{0}\right)$ and $q \in L^{p}\left(\Gamma_{-} ; \rho_{-}\right)$,

$$
\|u\|_{L^{p}\left(Q_{T}\right)} \leq\left(1-\frac{K}{\lambda}\right)^{-1} e^{\lambda T}\left(\left\|u_{0}\right\|_{L^{p}\left(\Omega \times U ; l_{0}\right)}+\|q\|_{L^{p}\left(\Gamma_{-} ; \rho_{-}\right)}\right)
$$

for $\lambda>K$.

We may abbreviate the estimate (1.7) as

$$
\|u\|_{L^{p}\left(Q_{T}\right)} \leq C\left(\left\|u_{0}\right\|_{L^{p}\left(\Omega \times U ; l_{0}\right)}+\|q\|_{L^{p}\left(\Gamma_{-} ; \rho_{-}\right)}\right)
$$

in order to show well-posedness of (1.1)-(1.3), but we prefer (1.7) in order to clarify dependent relations in constants appeared in the estimates, since we will aim at an inverse problem of (1.1)-(1.3) in future.

According to Ukai [11], the derivative of the weak solution along a characteristic line $\left(\frac{\partial u}{\partial l}\right)(t, x, \xi):=\left(\frac{\partial}{\partial t}+\xi \cdot \nabla_{x}\right) u(t, x, \xi)\left((t, x, \xi) \in Q_{T}\right)$ belongs to $L^{p}\left(Q_{T}\right)$, and it has the estimate

$$
\left\|\frac{\partial u}{\partial l}\right\|_{L^{p}\left(Q_{T}\right)} \leq K\|u\|_{L^{p}\left(Q_{T}\right)} .
$$

The estimate (1.7) follows from (1.8) and the fact that the weak solution satisfies the integral formula

$$
\begin{aligned}
u(t, x, \xi)= & u\left(t-l_{-}(t, x, \xi), x-l_{-}(t, x, \xi) \xi, \xi\right) \\
& +\int_{0}^{l_{-}(t, x, \xi)}\{-\mu(t-s, x-s \xi, \xi) u(t-s, x-s \xi, \xi) \\
& \left.+\int_{U} k\left(t-s, x-s \xi, \xi, \xi^{\prime}\right) u\left(t-s, x-s \xi, \xi^{\prime}\right) d \xi^{\prime}\right\} d s .
\end{aligned}
$$

Remark 1.1. We can minimize the constant in (1.7) as a function of the parameter $\lambda$, and $\min _{\lambda>K}\left(1-\frac{K}{\lambda}\right) e^{\lambda K}=(1+L) e^{L}$, where $L=\frac{1}{2} K T\left(1+\sqrt{1+\frac{4}{K T}}\right)$.

Let $u(t)$ be the weak solution to (1.1)-(1.3), and let $\tilde{u}(t)$ be the one with perturbation, and we will estimate $\|\tilde{u}(t)-u(t)\|_{L^{p}(\Omega \times U)}$ in the next section. We show, in $\S 3$, stability of the albedo operator under perturbation as an application of the main theorem stated in $\$ 2$. 
The transport equation is considered as the fundamental mathematical model to describe propagation of lights in biomedical tissue, and the scattering kernel, such as the HenyeyGreenstein kernel (see [3], [8]), is proposed through experimental data. We should remark that the mathematical model of the scattering kernel needs to admit observation errors, and we think it necessary to estimate their influence to the solutions before starting analysis of the inverse problem aimed at DOT; we expect our estimates to give a clue to guarantitative discussion on a priori information which is necessary in the analysis of the inverse problem.

\section{Estimates of the solutions under $L^{\infty}$-perturbation}

We give an estimate of the solutions $\|\tilde{u}(t)-u(t)\|_{L^{p}(\Omega \times U)}$, where we denote the solution to the perturbed system by $\tilde{u}$. The results mean stability of the $L^{p}$-weak solution under $L^{\infty}$ perturbation of the attenuation coefficient $\mu$ and of the scattering kernel $k$, and it implies difficulty in solving the inverse problem related with DOT. We may estimate $\|\tilde{u}(t)-u(t)\|_{L^{p}(\Omega \times U)}$ by the reduction of the errors caused by the perturbation to the inhomogeneous term of the equation (1.1), but we choose a direct calculation to clarify the constants in the final estimate (2.4).

Let us consider the following initial-boundary value problem:

$$
\begin{array}{rlrl}
\left(\frac{\partial}{\partial t}+\xi \cdot \nabla_{x}\right) \tilde{u}(t, x, \xi) & =-\tilde{\mu}(t, x, \xi) \tilde{u}(t, x, \xi)+\int_{U} \tilde{k}\left(t, x, \xi, \xi^{\prime}\right) \tilde{u}\left(t, x, \xi^{\prime}\right) d \xi^{\prime},(t, x, \xi) \in Q_{T}, \\
\tilde{u}(0, x, \xi) & =u_{0}(x, \xi), & x \in \Omega, \xi \in U, \\
\tilde{u}(t, x, \xi) & =q(t, x, \xi), & (t, x, \xi) \in \Gamma_{-},
\end{array}
$$

where $\tilde{\mu}(t, x, \xi) \in L^{\infty}\left(Q_{T}\right)$ and $\tilde{k}\left(t, x, \xi, \xi^{\prime}\right) \in L^{\infty}\left(Q_{T} \times U\right)$. The problem (2.1)-(2.3) is a perturbed system of (1.1)-(1.3), but we note that the initial data and the boundary data are the same. To state our estimate, we introduce constants $\tilde{K}$ and $\Delta K$ by

$$
\tilde{K}:=\|\tilde{\mu}\|_{L^{\infty}\left(Q_{T}\right)}+|U|\|\tilde{k}\|_{L^{\infty}\left(Q_{T} \times U\right)}, \quad \Delta K:=\|\tilde{\mu}-\mu\|_{L^{\infty}\left(Q_{T}\right)}+|U|\|\tilde{k}-k\|_{L^{\infty}\left(Q_{T} \times U\right)} .
$$

Theorem 2.1. Let $u \in L^{p}\left(Q_{T}\right)(1 \leq p<\infty)$ be the $L^{p}$-weak solution to (1.1)-(1.3), and let $\tilde{u} \in$ $L^{p}\left(Q_{T}\right)$ be that to (2.1)-(2.3). We have

$$
\|\tilde{u}(t)-u(t)\|_{L^{p}(\Omega \times U)} \leq 2^{1-\frac{1}{p}} \Delta K t^{1-\frac{1}{p}} \exp \left(\frac{2^{p-1} \tilde{K}^{p} t^{p}}{p^{2}}\right)\|u\|_{L^{p}\left(Q_{T}\right)} .
$$

Proof. We recall the integral equation (1.9) which the $L^{p}$-weak solution to (1.1)-(1.3) satisfies, and we obtain

$$
\tilde{u}(t, x, \xi)-u(t, x, \xi)=-\int_{0}^{l_{-}(t, x, \xi)}\{\tilde{\mu}(t-s, x-s \xi, \xi) \tilde{u}(t-s, x-s \xi, \xi)
$$




$$
\begin{gathered}
-\mu(t-s, x-s \xi, \xi) u(t-s, x-s \xi, \xi)\} d s \\
+\int_{0}^{l_{-}(t, x, \xi)}\left\{\int _ { U } \left\{\tilde{k}\left(t-s, x-s \xi, \xi, \xi^{\prime}\right) \tilde{u}\left(t-s, x-s \xi, \xi^{\prime}\right)\right.\right. \\
\left.\left.-k\left(t-s, x-s \xi, \xi, \xi^{\prime}\right) u\left(t-s, x-s \xi, \xi^{\prime}\right)\right\} d \xi^{\prime}\right\} d s .
\end{gathered}
$$

We are going to give the proof of (2.4) in two steps.

(1) 1st step: estimation of $\|\tilde{u}(t)-u(t)\|_{L^{p}(\Omega \times U)}$.

From (2.5), we have

$$
\|\tilde{u}(t)-u(t)\|_{L^{p}(\Omega \times U)} \leq\left\|\int_{0}^{l_{-}(t, \cdot, \cdot)}(\tilde{\mu} \tilde{u}-\mu u) d s\right\|_{L^{p}(\Omega \times U)}+\left\|\int_{0}^{l_{-}(t, \cdot, \cdot)}\left(\int_{U}(\tilde{k} \tilde{u}-k u) d \xi^{\prime}\right) d s\right\|_{L^{p}(\Omega \times U)} .
$$

Since

$$
\left\|\int_{0}^{l_{-}}(\tilde{\mu} \tilde{u}-\mu u) d s\right\|_{L^{p}} \leq\|\tilde{\mu}\|_{L^{\infty}}\left\|\int_{0}^{l_{-}}|\tilde{u}-u| d s\right\|_{L^{p}}+\|\tilde{\mu}-\mu\|_{L^{\infty}}\left\|\int_{0}^{l_{-}}|u| d s\right\|_{L^{p}},
$$

we estimate the first term of the right hand side of (2.6) as follows;

$$
\begin{aligned}
& \left\|\int_{0}^{l_{-}(t, \cdot, \cdot)}(\tilde{\mu} \tilde{u}-\mu u) d s\right\|_{L^{p}(\Omega \times U)} \\
& \leq\|\tilde{\mu}\|_{L^{\infty}}\left(\int_{\Omega \times U}\left(\int_{0}^{l_{-}(t, x, \xi)}|\tilde{u}-u| d s\right)^{p} d x d \xi\right)^{\frac{1}{p}}+\|\tilde{\mu}-\mu\|_{L^{\infty}}\left(\int_{\Omega \times U}\left(\int_{0}^{l_{-}(t, x, \xi)}|u| d s\right)^{p} d x d \xi\right)^{\frac{1}{p}} \\
& \leq\|\tilde{\mu}\|_{L^{\infty}}\left(\int_{\Omega \times U} l_{-}^{p-1}\left(\int_{0}^{l_{-}(t, x, \xi)}|\tilde{u}-u|^{p} d s\right) d x d \xi\right)^{\frac{1}{p}} \\
& \quad+\|\tilde{\mu}-\mu\|_{L^{\infty}}\left(\int_{\Omega \times U} l_{-}^{p-1}\left(\int_{0}^{l_{-}(t, x, \xi)}|u|^{p} d s\right) d x d \xi\right)^{\frac{1}{p}} \\
& \leq\|\tilde{\mu}\|_{L^{\infty}} t^{1-\frac{1}{p}}\left(\int_{0}^{t}\left\|\tilde{u}\left(t^{\prime}, \cdot, \cdot\right)-u\left(t^{\prime}, \cdot, \cdot\right)\right\|_{L^{p}(\Omega \times U)}^{p} d t^{\prime}\right)^{\frac{1}{p}}+\|\tilde{\mu}-\mu\|_{L^{\infty}} t^{1-\frac{1}{p}}\|u\|_{L^{p}\left(Q_{T}\right)} \cdot
\end{aligned}
$$

We recall $0 \leq l_{-}(t, x, \xi) \leq t \leq T$, and we remark the above estimation is justified by the FubiniTonelli theorem. By the same manner, the second term is estimated as

$$
\begin{aligned}
& \left\|\int_{0}^{l_{-}(t, \cdot, \cdot)}\left(\int_{U}(\tilde{k} \tilde{u}-k u) d \xi^{\prime}\right) d s\right\|_{L^{p}(\Omega \times U)} \leq\left\{\int_{\Omega \times U} l_{-}^{p-1}\left(\int_{0}^{l_{-}}\left|\int_{U} \tilde{k}(\tilde{u}-u) d \xi^{\prime}\right|^{p} d s\right) d x d \xi\right\}^{\frac{1}{p}} \\
& \quad+\left\{\int_{\Omega \times U} l_{-}^{p-1}\left(\int_{0}^{l_{-}}\left|\int_{U}(\tilde{k}-k) u d \xi^{\prime}\right|^{p} d s\right) d x d \xi\right\}^{\frac{1}{p}} \\
& \leq|U|^{1-\frac{1}{p}} t^{1-\frac{1}{p}}\|\tilde{k}\|_{L^{\infty}}\left(\int_{U} \int_{0}^{t}\left\|\tilde{u}\left(t^{\prime}, \cdot, \cdot\right)-u\left(t^{\prime}, \cdot, \cdot\right)\right\|_{L^{p}(\Omega \times U)}^{p} d t^{\prime} d \xi^{\prime}\right)^{\frac{1}{p}} \\
& +|U|^{1-\frac{1}{p}} t^{1-\frac{1}{p}}\|\tilde{k}-k\|_{L^{\infty}}\left(\int_{U} \int_{0}^{t}\left\|u\left(t^{\prime}, \cdot, \cdot\right)\right\|_{L^{p}(\Omega \times U)}^{p} d t^{\prime} d \xi^{\prime}\right)^{\frac{1}{p}}
\end{aligned}
$$




$$
\leq|U| t^{1-\frac{1}{p}}\|\tilde{k}\|_{L^{\infty}}\left(\int_{0}^{t}\left\|\tilde{u}\left(t^{\prime}, \cdot, \cdot\right)-u\left(t^{\prime}, \cdot, \cdot\right)\right\|_{L^{p}(\Omega \times U)}^{p} d t^{\prime}\right)^{\frac{1}{p}}+|U|\|\tilde{k}-k\|_{L^{\infty}} t^{1-\frac{1}{p}}\|u\|_{L^{p}\left(Q_{T}\right)}
$$

Since we know $(a+b)^{p} \leq 2^{p-1}\left(a^{p}+b^{p}\right)$ for $a>0, b>0$, we conclude, from (2.7) and (2.8),

$$
\|\tilde{u}(t)-u(t)\|_{L^{p}(\Omega \times U)}^{p} \leq t^{p-1} 2^{p-1} \tilde{K}^{p}\left(\int_{0}^{t}\left\|\tilde{u}\left(t^{\prime}\right)-u\left(t^{\prime}\right)\right\|_{L^{p}(\Omega \times U)}^{p} d t^{\prime}+\frac{\Delta K^{p}}{\tilde{K}^{p}}\|u\|_{L^{p}\left(Q_{T}\right)}^{p}\right) .
$$

(2) 2nd step: Gronwall type estimation.

Let us define a function $F(t)$ by

$$
F(t):=\int_{0}^{t}\left\|\tilde{u}\left(t^{\prime}\right)-u\left(t^{\prime}\right)\right\|_{L^{p}(\Omega \times U)}^{p} d t^{\prime}+\frac{\Delta K^{p}}{\tilde{K}^{p}}\|u\|_{L^{p}\left(Q_{T}\right)}^{p},
$$

then we have, from (2.9), $\frac{d F}{d t} \leq 2^{p-1} \tilde{K}^{p} t^{p-1} F$. Hence we have

$$
\frac{d}{d t}\left(\exp \left(-\frac{2^{p-1} \tilde{K}^{p}}{p} t^{p}\right) F(t)\right) \leq 0
$$

and immediately we obtain

$$
\|\tilde{u}(t)-u(t)\|_{L^{p}(\Omega \times U)}^{p}=\frac{d F}{d t} \leq 2^{p-1} \tilde{K}^{p} t^{p-1} \exp \left(\frac{2^{p-1} \tilde{K}^{p}}{p} t^{p}\right) F(0) .
$$

Thus we obtain the estimate (2.4)

Remark 2.2. Substitution of the estimate $\|u\|_{L^{p}\left(Q_{T}\right)}$ of (1.7) into (2.4) leads us to the estimate

$$
\begin{aligned}
\|\tilde{u}(t)-u(t)\|_{L^{p}(\Omega \times U)} \leq & 2^{1-\frac{1}{p}} \Delta K t^{1-\frac{1}{p}} \exp \left(\frac{2^{p-1} \tilde{K}^{p} t^{p}}{p^{2}}\right) \\
& \times\left(1-\frac{K}{\lambda}\right)^{-1} e^{\lambda T}\left(\left\|u_{0}\right\|_{L^{p}\left(\Omega \times U ; l_{0}\right)}+\|q\|_{L^{p}\left(\Gamma_{-} ; \rho_{-}\right)}\right) .
\end{aligned}
$$

\section{Stability estimate for the Albedo operator}

Applied mathematicians ([9], [4], [12] etc.) have defined the Albedo operator for the transport equation and have discussed inverse problems, and we also give an estimate to the operator under the same perturbation as in $\$ 2$. Let us define the Albedo operator for the transport equation with the homogeneous Cauchy data. Before starting discussion, we introduce the Banach space $L^{p}\left(\Gamma_{+} ; \rho_{+}\right)$in the same manner with $L^{p}\left(\Gamma_{-} ; \rho_{-}\right)$, which is defined in $\S 1$.

Since we have (1.8), we can introduce a Banach space $W^{p}\left(Q_{T}\right)$ by

$$
W^{p}\left(Q_{T}\right):=\left\{u \in L^{p}\left(Q_{T}\right) \mid \frac{\partial u}{\partial l} \in L^{p}\left(Q_{T}\right)\right\}
$$


with the norm $\|u\|_{W^{p}\left(Q_{T}\right)}:=\|u\|_{L^{p}\left(Q_{T}\right)}+T\left\|\frac{\partial u}{\partial l}\right\|_{L^{p}\left(Q_{T}\right)}$, and we note that the weak solution to (1.1)-(1.3) belongs to $W^{p}\left(Q_{T}\right)$. Let us define the trace operator $\gamma_{ \pm}: W^{p}\left(Q_{T}\right) \rightarrow L^{p}\left(\Gamma_{ \pm} ; \rho_{ \pm}\right)$as follows. For $\phi \in C_{0}^{\infty}\left(\overline{Q_{T}}\right)$, integration along the characteristic line gives

$$
\phi\left(t-l_{-}(t, x, \xi), x-\xi l_{-}(t, x, \xi), \xi\right)=\int_{0}^{l_{-}(t, x, \xi)} \frac{\partial \phi}{\partial l}(t-s, x-s \xi, \xi) d s+\phi(t, x, \xi),
$$

which leads us to $\left\|\left.\phi\right|_{\Gamma_{-}}\right\|_{L^{p}\left(\Gamma_{-} ; \rho_{-}\right)} \leq\|\phi\|_{W^{p}\left(Q_{T}\right)}$. Hence we can define the trace operator $\gamma_{-}$as continuous extention of the restriction operator $\left.\right|_{\Gamma_{-}}$. It is quite the same for $\gamma_{+}$. We remark, from (1.3), that $\gamma_{-} u=q$ for the weak solution $u$, and we note that the trace $\gamma_{+} u$ belongs to $L^{p}\left(\Gamma_{+} ; \rho_{+}\right)$, since we have $u \in W^{p}\left(Q_{T}\right)$. Thus we define the Albedo operator.

Definition 3.1. (Albedo operator)

Let $u \in L^{p}\left(Q_{T}\right)$ be the weak solution to (1.1)-(1.3) with the homogeneous Cauchy data; $u_{0}(x, \xi)=0$ in (1.2). We define an operator $A: L^{p}\left(\Gamma_{-} ; \rho_{-}\right) \rightarrow L^{p}\left(\Gamma_{+} ; \rho_{+}\right)$by $A q:=\gamma_{+} u$, and we call it the Albedo operator.

Since we assume $u_{0}(x, \xi)=0$, we have, from (1.8) and Proposition 1.2,

$$
\|A q\|_{L^{p}\left(\Gamma_{+} ; \rho_{+}\right)} \leq\|u\|_{W^{p}\left(Q_{T}\right)} \leq(1+K T)\left(1-\frac{K}{\lambda}\right)^{-1} e^{\lambda T}\|q\|_{L^{P}\left(\Gamma_{-} ; \rho_{-}\right)}
$$

for $\lambda>K$ and the albedo operator is well-defined as a bounded operator. We state our result.

Theorem 3.1. Suppose $1 \leq p<\infty$. Let $A$ be the albedo operator connected with (1.1)-(1.3), and let $\tilde{A}$ be the one connected with (2.1)-(2.3). Then we have

$$
\|\tilde{A}-A\|_{B\left(L^{p}\left(\Gamma_{-} ; \rho_{-}\right), L^{p}\left(\Gamma_{+} ; \rho_{+}\right)\right)} \leq\left(1-\frac{K}{\lambda}\right)^{-1} e^{\lambda T} \Delta K\left\{\frac{1+\tilde{K} T}{\tilde{K}}\left(\exp \left(\frac{(2 \tilde{K} T)^{p}}{2 p}\right)-1\right)^{\frac{1}{p}}+T\right\}
$$

for $\lambda>K$.

Proof. We firstly remark, by integrating the both side of (2.4) that

$$
\|\tilde{u}-u\|_{L^{p}\left(Q_{T}\right)} \leq \frac{\Delta K}{\tilde{K}}\left(\exp \left(\frac{2^{p-1} \tilde{K}^{p}}{p} t^{p}\right)-1\right)^{\frac{1}{p}}\|u\|_{L^{p}\left(Q_{T}\right)}
$$

Since we assume $u_{0}(x, \xi)=0$ in (1.2) and (2.2), we have

$$
\left(\frac{\partial}{\partial t}+\xi \cdot \nabla_{x}\right)(\tilde{u}-u)=-\tilde{\mu}(\tilde{u}-u)+\int_{U} \tilde{k}(\tilde{u}-u) d \xi^{\prime}-(\tilde{\mu}-\mu) u+\int_{U}(\tilde{k}-k) u d \xi^{\prime},
$$

and

$$
\begin{aligned}
\|\tilde{A} q-A q\|_{L^{p}\left(\Gamma_{+} ; \rho_{+}\right)} & \leq\|\tilde{u}-u\|_{W^{p}\left(Q_{T}\right)}=\|\tilde{u}-u\|_{L^{p}\left(Q_{T}\right)}+T\left\|\frac{\partial \tilde{u}}{\partial l}-\frac{\partial u}{\partial l}\right\|_{L^{p}\left(Q_{T}\right)} \\
& \leq\|\tilde{u}-u\|_{L^{p}\left(Q_{T}\right)}+\tilde{K} T\|\tilde{u}-u\|_{L^{p}\left(Q_{T}\right)}+\Delta K T\|u\|_{L^{p}\left(Q_{T}\right)} .
\end{aligned}
$$


Substituting the estimates (1.7) and (3.2), we conclude

$$
\begin{aligned}
\|\tilde{A} q-A q\|_{L^{p}\left(\Gamma_{+} ; \rho_{+}\right)} & \leq(1+\tilde{K} T) \frac{\Delta K}{\tilde{K}}\left(\exp \left(\frac{2^{p-1} \tilde{K}^{p}}{p} T^{p}\right)-1\right)^{\frac{1}{p}}\|u\|_{L^{p}\left(Q_{T}\right)}+\Delta K T\|u\|_{L^{p}\left(Q_{T}\right)} \\
& \leq\left(1-\frac{K}{\lambda}\right)^{-1} e^{\lambda T}\|q\|_{L^{p}\left(\Gamma_{-} ; \rho_{-}\right)} \Delta K\left\{\frac{1+\tilde{K} T}{\tilde{K}}\left(\exp \left(\frac{(2 \tilde{K} T)^{p}}{2 p}\right)-1\right)^{\frac{1}{p}}+T\right\} .
\end{aligned}
$$

Hence we obtain our final estimate.

\section{Acknowledgement}

This work is partially supported by Grant-in-Aid for Challenging Exploratory Research 21654016. Some of the results in this paper were orally presented at Taiwan-Japan Joint Workshop on Inverse Problems held in 2010 at National Taiwan University.

\section{References}

[1] P. Arianfar and H. Emamirad, Relation between scattering and albedo operators in linear transport theory, Transport Theory and Stat. Physics 23 (1994), 517-531.

[2] S. R. Arridge and J. C. Hebden, Optical imaging in medicine: II. Modelling and reconstruction, Phys. Med. Biol. 42 (1997), 841-853.

[3] S. R. Arridge, Optical tomography in medical imaging, Inverse Problems 15(1999), R41-R93.

[4] M. Choulli and P. Stefanov, An inverse scattering and inverse boundary value problems for the linear Boltzmann equation, Comm. Partial Diff. Equ. 21 (1996), 763-785.

[5] A. Douglis, The solution of multidimensional generalized transport equation and their calculation by difference methods, Numerical Solutions of Partial Differential Equations (J. H. Bramble. Ed.), Academic Press, New-York, 1966.

[6] H. Emamirad and V. Protopopescu, Relationship between the albedo and scattering operators for the Boltzmann equation with semi-transparent boundary conditions, Math. Meth. Appl. Sci. 19 (1996), 1-13.

[7] A. H. Hielscher, R. E. Alcouffe and R. L. Barbour, Comparison of finite-difference transport and diffusion calculations for photon migration in homogeneous and heterogeneous tissues, Phys. Med. Biol. 43 (1998), 12851302.

[8] A. D. Klose and E. W. Larsen, Light transport in biological tissue based on the simplified spherical harmonics equations, J. Comput. Phys. 220 (2006), 441-470.

[9] F. Monard and G. Bal, An accurate solver for forward and inverse transport, J. Comp. Phys. 229 (2010), 49524979.

[10] P. Stefanov and G. Uhlmann, Optical Tomography in two dimensions, Methods Appl. Anal. 10 (2003), 1-9.

[11] S. Ukai, Transport Equation (Japanese), Sangyo-Tosho Publishing, Tokyo, 1976.

[12] J. N. Wang, Stability estimates of an inverse problem for the stationary transport equation, Ann. Inst. Henri Poincaré 70 (1999), 473-495.

Graduate School of Informatics, Kyoto University, Yoshida-Honmachi, Kyoto, 606-8501, Japan.

E-mail: daiki596@acs.i.kyoto-u.ac.jp

Graduate School of Economics, Hitotsubashi University, Kunitachishi-Naka 2-1, Tokyo, 186-8601, Japan.

E-mail: higashim@econ.hit-u.ac.jp

Graduate School of Informatics, Kyoto University, Yoshida-Honmachi, Kyoto, 606-8501, Japan.

E-mail: iso@acs.i.kyoto-u.ac.jp 